\title{
Saudi Radiology Personnel's Perceptions of Artificial Intelligence Implementation: A Cross-Sectional Study
}

\author{
Abdulaziz A Qurashi ${ }^{1}$ \\ Rashed K Alanazi' \\ Yasser M Alhazmi' \\ Ahmed S Almohammadi ${ }^{1}$ \\ Walaa M Alsharif ${ }^{\prime}$ \\ Khalid M Alshamrani $\mathbb{D}^{2,3}$ \\ 'Diagnostic Radiology Technology \\ Department, College of Applied Medical \\ Sciences, Taibah University, Madinah, \\ Saudi Arabia; ${ }^{2}$ College of Applied Medical \\ Sciences, King Saud bin Abdulaziz \\ University for Health Sciences, Jeddah, \\ Saudi Arabia; ${ }^{3}$ King Abdullah \\ International Medical Research Center, \\ Jeddah, Saudi Arabia
}

Purpose: Artificial intelligence (AI) in radiology has been a subject of heated debate. The external perception is that algorithms and machines cannot offer better diagnosis than radiologists. Reluctance to implement AI maybe due to the opacity in how AI applications work and the challenging and lengthy validation process. In this study, Saudi radiology personnel's familiarity with AI applications and its usefulness in clinical practice were investigated.

Methods: A cross-sectional study was conducted in Saudi Arabia among radiology personnel from March to April 2021. Radiology personnel nationwide were surveyed electronically using Google form. The questionnaire included 12-questions related to AI usefulness in clinical practice and participants' knowledge about AI and their acceptance level to learn and implement this technology into clinical practice. Participants' trust level was also measured; Kruskal-Wallis test was used to examine differences between groups.

Results: A total of 224 respondents from various radiology-related occupations participated in the survey. The lowest trust level in AI applications was shown by radiologists $(p=0.033)$. Eighty-two percent of participants $(n=184)$ had never used AI in their departments. Most respondents $(n=160,71.4 \%)$ reported lack of formal education regarding AI-based applications. Most participants $(n=214,95.5 \%)$ showed strong interest in AI education and are willing to incorporate it into the clinical practice of radiology. Almost half of radiography students $(22 / 46$, $47.8 \%)$ believe that their job might be at risk due to AI application $(\mathrm{p}=0.038)$.

Conclusion: Radiology personnel's knowledge of AI has a significant impact on their willingness to learn, use and adapt this technology in clinical practice. Participants demonstrated a positive attitude towards AI, showed a reasonable understanding and are highly motivated to learn and incorporate it into clinical practice. Some participants felt that their jobs were threatened by AI adaptation, but this belief might change with good training and education programmes.

Keywords: artificial intelligence, AI-based applications, radiology, radiologists, imaging modalities

\section{Introduction}

Medical imaging has improved and become more accessible, increasing the radiology reporting workload in hospitals and clinics worldwide. The higher demand has primarily been seen in imaging modalities that are time-consuming, such as computed tomography (CT) and magnetic resonance imaging (MRI). ${ }^{1}$ Therefore, radiologists will be challenged to handle an overwhelming number of cases and report them in a timely manner. ${ }^{2}$ Over the last few years, artificial intelligence (AI) applications have become a subject of heated debate in radiological field. ${ }^{3} \mathrm{AI}$ is one of the distinct fields of the discipline of computer science that can simulate the
Correspondence: Abdulaziz A Qurashi Diagnostic Radiology Technology, Taibah University, Madinah, 42353, Saudi Arabia Tel +966 148618888 Ext. 3603

Email aaqurashi@taibahu.edu.sa 
cognitive functions of humans such as problem solving, in which the computer can perform a very complex task with high accuracy. ${ }^{4}$ Although some of the radiologist's tasks may be complicated for AI-based applications, the practice of radiologists can improve since the performance of tedious and routine tasks and reading time can be achieved more efficiently with the help of AI-based applications., ${ }^{4,5}$ Reduction in reading time was observed in detecting pulmonary metastasis by suppressing vessels on $\mathrm{CT}$ thorax imaging. ${ }^{6}$ Several additional studies have shown that automated quantification of nodules, bone age prediction, and knee assessment (eg, osteoarthritis) can help reduce radiologists' workload and improve diagnosis accuracy. ${ }^{7-9}$ Additionally, AI-based applications may improve image interpretation in differentiating lung viruses such as COVID-19 from other pneumonia diseases. ${ }^{10,11}$

It remains to be seen whether AI will aid radiographers in clinical decision-making, such as scanning protocols or radiation doses. A study suggested that AI is poised to enhance the role of radiographers within radiology departments (eg, pre-examination assessment, examination planning, image processing). ${ }^{12}$ Automation in radiography, however, might limit the radiographer's role and contribution on decision-making. ${ }^{12,13}$

Several tasks can be provided using AI, including processing, reporting, perception, and reasoning. ${ }^{14}$ Perception and reasoning tasks can be divided into several functionalities including segmentation where boundaries of organs are designated. Extraction is the next step where unwanted organs in the image are isolated. Detecting and highlighting a specific abnormality and comparing it with patient's images to evaluate the changes over time. A few AI applications are also available to predict the prognosis (ie, predict the course of the disease). ${ }^{15}$ There are several AI strengths highlighted in the literature; however, limitations of the existing prediction models have also been noted due to the lack of data and concerns with validation and promotion (Table 1). ${ }^{16,17}$

Several studies in the literature showed that AI-based applications will not replace radiologist's role; in fact, it will improve radiology services and radiologists' performance. ${ }^{18}$ However, other researchers were worried that AI-based applications could be influencing medical students' decisions from choosing radiology as a profession. ${ }^{19}$ Thus, the purpose of this study was to evaluate and contrast the current level of knowledge and perceptions among different categories of radiology staff in Saudi Arabia regarding the current and future AI applications and to describe future requirements for successful implementation.

\section{Methodology Study Design}

A cross-sectional, survey-based study was conducted among radiologists, radiographers, clinical application specialists and internship radiography students across Saudi Arabia using a non-probability convenience sampling technique. An online questionnaire was distributed among participants via Google Form for the period of March to April 2021. The questionnaire was designed by the research team. Initially, the study was piloted with four academic lecturers and two radiologists, and corrections were made based on the comments received.

Demographic background information about participants' gender, years of experience, place of work, type institution and occupational was collected. The questionnaire contained eight closed questions (ie, yes/no) related to participants' familiarity with $\mathrm{AI}$ and its current

Table I Strength and Limitations of Artificial Intelligence in Radiology

\begin{tabular}{|c|c|}
\hline Strengths & Limitations \\
\hline $\begin{array}{l}\text { - Automated lesions screening, detection, segmentation, and characterization } \\
\text { by using input data from other modalities (eg, x-ray, CT, MRI). }\end{array}$ & $\begin{array}{l}\text { - Al-based applications not familiar with the global context of } \\
\text { patients. }\end{array}$ \\
\hline - Classify images based on the presence or absence of abnormality. & - Training data time, cost, and resource consuming. \\
\hline - Extract additional data from previous detected abnormality (eg, lesion) & - Lack the power of supervised algorithms. \\
\hline $\begin{array}{l}\text { - Identification of anatomical landmarks or organs, which are important for } \\
\text { both image acquisition and analysis. }\end{array}$ & $\begin{array}{l}\text { - Lack of accurate validation of the } \mathrm{Al} \text { applications during training } \\
\text { which may lead to random noise than the actual data. }\end{array}$ \\
\hline $\begin{array}{l}\text { - Detecting scan planes for rapid examination planning and minimum } \\
\text { interindividual variability, bias and scanning time. }\end{array}$ & $\begin{array}{l}\text { - Lack of specific multidisciplinary road maps for Al-based } \\
\text { application implementation in medical imaging field. }\end{array}$ \\
\hline
\end{tabular}


application to assess radiology personnel current knowledge in relation to the $\mathrm{AI}$ and its application in radiology.

The questionnaire contained additional four questions, which aimed to assess the level of agreement concerning the usefulness of AI-based applications in clinical practice. Participants were asked to score these four questions in a 5-point Likert scale (ie, $1=$ strongly agree, 2 = agree, $3=$ neither agree nor disagree, $4=$ disagree, $5=$ strongly disagree).

\section{Ethical Consideration}

An ethical approval has been obtained from the institutional review board in College of Applied Medical Science at Taibah University (Reference Number: 2020/85/314/ RAD). Participation in this study was voluntary and informed consent was obtained.

\section{Statistical Analyses}

Statistical analysis was performed using the IBM statistical package for social sciences (SPSS version 20, PASW, Chicago, IL). Kruskal-Wallis one-way analysis of variance test was used to examine differences between groups. Demographical characteristic analysis was performed for the whole study sample. A descriptive analysis of data was carried out using counts and percentages. A p-value of $\leq 0.05$ was considered significant.

\section{Results}

The study sample included 224 respondents (61.6\% males and $38.4 \%$ females); of which 120 (53.6\%) were radiographers, $46(20.5 \%)$ were internship radiography students, $40(17.9 \%)$ were radiologists, while $18(8 \%)$ were clinical application specialists. The demographic characteristics of the participants, such as age, years of experience and the departments are shown in Table 2 and Figure 1. The perceptions and knowledge level of different radiology personnel in Saudi Arabia towards the current and future AI applications were analysed based on participants' profession as shown in Table 3.

Overall, most of the participants $(n=186,83 \%)$ were familiar with the machine learning function and AI concept. Among the other three groups, radiologists had the most familiarity $(\mathrm{p}=0.001)$. However, the results indicated that the lowest trust level in AI applications was shown by radiologists $(p=0.033)$.
Table 2 Demographic Characteristic of Study Sample

\begin{tabular}{|c|c|c|}
\hline \multicolumn{2}{|r|}{ Demographic } & $\mathbf{N}(\%)$ \\
\hline \multirow[t]{5}{*}{ Age } & $<25$ years & $88(39.3 \%)$ \\
\hline & $25-34$ years & $82(36.6 \%)$ \\
\hline & $35-44$ years & $32(14.3 \%)$ \\
\hline & $45-54$ years & $20(8.9 \%)$ \\
\hline & $>55$ years & $2(0.9 \%)$ \\
\hline \multirow{5}{*}{$\begin{array}{l}\text { Years of } \\
\text { experience }\end{array}$} & $<3$ years & $84(37.5 \%)$ \\
\hline & $3-5$ years & $24(10.7 \%)$ \\
\hline & $6-10$ years & $32(14.3 \%)$ \\
\hline & $>10$ years & $46(20.5 \%)$ \\
\hline & Never & $38(17 \%)$ \\
\hline \multirow[t]{9}{*}{ Departments } & General radiography & $56(25 \%)$ \\
\hline & Computed Tomography (CT) & 48 (2I.4\%) \\
\hline & Magnetic Resonance Imaging (MRI) & $24(10.7 \%)$ \\
\hline & Nuclear Medicine (NM) & $14(6.3)$ \\
\hline & Ultrasound (US) & $26(11.6 \%)$ \\
\hline & $\begin{array}{c}\text { Picture archiving and communication } \\
\text { system (PACS) }\end{array}$ & $2(0.9 \%)$ \\
\hline & Interventional radiology & $8(3.6 \%)$ \\
\hline & Administration & $6(2.7 \%)$ \\
\hline & Other & $40(17.8 \%)$ \\
\hline \multirow[t]{4}{*}{ Qualifications } & Diploma (Dip) & $12(5.4 \%)$ \\
\hline & Bachelor (BSc) & $150(67 \%)$ \\
\hline & Master (MSc) & $24(10.6 \%)$ \\
\hline & Doctor of Philosophy (PhD) & $38(17 \%)$ \\
\hline \multirow[t]{4}{*}{ Occupation } & Radiographers & $120(53.6 \%)$ \\
\hline & Radiologists & 40 (I7.9\%) \\
\hline & Clinical application specialists & $18(8 \%)$ \\
\hline & Internship radiography students & $46(20.5 \%)$ \\
\hline \multicolumn{2}{|l|}{ Total } & 224 \\
\hline
\end{tabular}

In response to the question related to the use of $\mathrm{AI}$ in participants' departments, $82.1 \%(n=184)$ of the participants had never used it. Furthermore, the majority of respondents mentioned the lack of formal education and knowledge related to AI-based applications.

Most of the participants $(\mathrm{n}=214 ; 95.5 \%)$ showed high interest in using AI-based application in clinical practice 


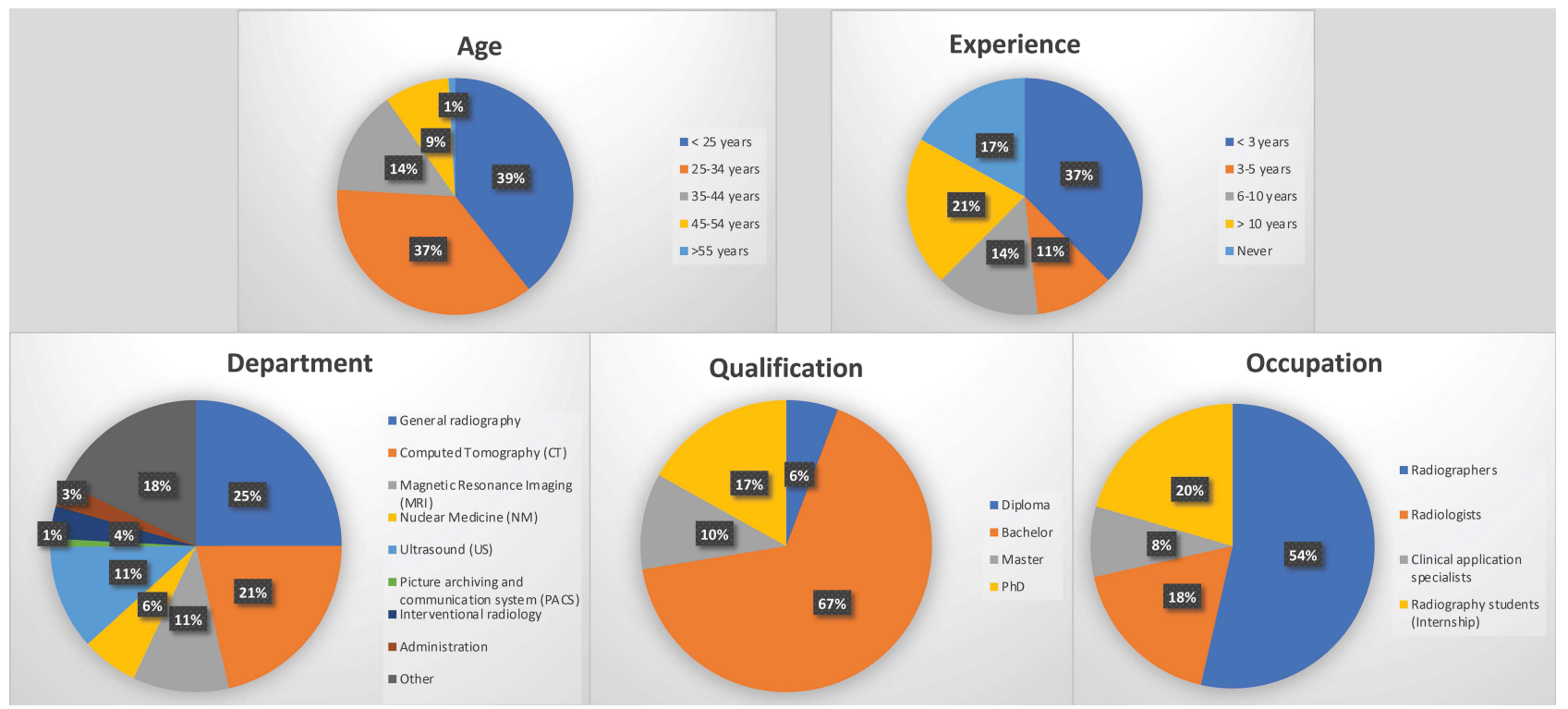

Figure I Demographic profile of the study participants.

(Table 3). While $92.9 \%$ of the participants showed willingness towards introducing $\mathrm{AI}$ in clinical practice of radiology.

For the question on whether AI will replace the participants' jobs, the answers showed that almost half of the students believed that their job is in danger due to this application $(\mathrm{p}=0.038)$.

The participants' level of agreement regarding the usefulness of AI-based application in clinical practice is listed in Table 4. The percentage of participants who either agree or strongly agree that $\mathrm{AI}$ is useful in clinical decision making such as justification of examination is $69.7(\mathrm{n}=156)$. Similarly, $66.1 \%(\mathrm{n}=148)$ of participants agree or strongly agree that $\mathrm{AI}$ is useful in automated imaging protocol selection according to clinical question and patient condition; whereas $75.1 \%(\mathrm{n}=168)$ and $69.6 \%(n=156)$ of participants agree or strongly agree that AI will be useful in improving diagnosis and saving time and will assist in personalizing imaging for patients

Table 3 Participants' Responses to Artificial Intelligence Survey

\begin{tabular}{|c|c|c|c|c|c|c|c|c|c|c|}
\hline \multirow[b]{3}{*}{ N (\%) } & \multirow{2}{*}{\multicolumn{2}{|c|}{ Total }} & \multicolumn{8}{|c|}{ Profession } \\
\hline & & & \multicolumn{2}{|c|}{ Radiologists } & \multicolumn{2}{|c|}{ Radiographers } & \multicolumn{2}{|c|}{$\begin{array}{c}\text { Radiography } \\
\text { Students }\end{array}$} & \multicolumn{2}{|c|}{$\begin{array}{l}\text { Clinical Application } \\
\text { Specialists }\end{array}$} \\
\hline & Yes & No & Yes & No & Yes & No & Yes & No & Yes & No \\
\hline QI* & 200 (89.3\%) & $24(10.7 \%)$ & 34 (85\%) & $6(15 \%)$ & 108 (90\%) & 12 (10\%) & 44 (95.7\%) & 2 (4.3\%) & I4 (77.8\%) & 4 (22.2\%) \\
\hline Q2* & I54 (68.8\%) & 70 (3I.2\%) & 22 (55\%) & I8 (45\%) & 92 (76.7\%) & 28 (23.3\%) & 30 (65.2\%) & 16 (34.8\%) & $10(55.6 \%)$ & 8 (44.4\%) \\
\hline Q3* & I86 (83\%) & 38 (17\%) & 40 (100\%) & - & 98 (81.7\%) & 22 (I8.3\%) & 38 (82.6\%) & $8(17.4 \%)$ & $10(55.6 \%)$ & $8(44.4 \%)$ \\
\hline Q4* & 40 (17.9\%) & I84 (82.1\%) & $10(25 \%)$ & $30(75 \%)$ & 24 (20\%) & $96(80 \%)$ & - & $46(100 \%)$ & $6(33.3 \%)$ & $12(66.7 \%)$ \\
\hline Q5* & 64 (28.6\%) & 160 (7I.4\%) & $12(30 \%)$ & $28(70 \%)$ & $32(26.7 \%)$ & 88 (73.3\%) & $10(21.7 \%)$ & 36 (78.3\%) & $10(55.6 \%)$ & 8 (44.4\%) \\
\hline Q6* & 214 (95.5\%) & 10 (4.5\%) & 38 (95\%) & $2(5 \%)$ & II 4 (95\%) & $6(5 \%)$ & 44 (95.7\%) & 2 (4.3\%) & $18(100 \%)$ & - \\
\hline Q7* & 72 (32.1\%) & 152 (67.9\%) & $12(30 \%)$ & $28(70 \%)$ & 32 (26.7\%) & 88 (73.3\%) & 22 (47.8\%) & 24 (52.2\%) & $6(33.3 \%)$ & 12 (66.7\%) \\
\hline Q8* & 208 (92.9\%) & $16(7.1 \%)$ & 38 (95\%) & $2(5 \%)$ & 108 (90\%) & $12(10 \%)$ & 44 (95.7\%) & $2(4.3 \%)$ & I8 (100\%) & - \\
\hline
\end{tabular}

Notes: QI*: Do you work freely with technology?, Q2*: Do you trust machine learning ability in analysing data for decision making purposes?, Q3*: Are you familiar that Al is the ability of machines to simulate the analytic functions of humans?, Q4*: Do you use Al tool in your department?, Q5*: Did you receive any formal education in any aspect of Al?, Q6*: Are you interested in learning about Al application?, Q7*: Do you think that Al will replace your job?, Q8*: Are you in favour of introducing Al in radiology practice. 
Table 4 Level of Agreement Concerning the Usefulness of Al-Based Applications in Clinical Practice

\begin{tabular}{|c|c|c|c|c|c|c|c|c|}
\hline & $\begin{array}{c}\text { Strongly } \\
\text { Agree } \\
\text { N (\%) } \\
\text { Code = I }\end{array}$ & $\begin{array}{l}\text { Agree } \\
\text { N (\%) } \\
\text { Code } \\
=2\end{array}$ & $\begin{array}{l}\text { Neither } \\
\text { Agree Nor } \\
\text { Disagree } \\
\text { N (\%) Code } \\
\quad=3\end{array}$ & $\begin{array}{l}\text { Disagree } \\
\text { N (\%) } \\
\text { Code }=4\end{array}$ & $\begin{array}{c}\text { Strongly } \\
\text { Disagree } \\
\text { N (\%) } \\
\text { Code = } 5\end{array}$ & $\begin{array}{c}95 \% \\
\text { Confidence } \\
\text { Interval }\end{array}$ & Median & Total \\
\hline $\begin{array}{l}\mathrm{Al} \text { is useful in clinical decision } \\
\text { making such as justification of } \\
\text { examination }\end{array}$ & $\begin{array}{c}60 \\
(26.8 \%)\end{array}$ & $\begin{array}{c}96 \\
(42.9 \%)\end{array}$ & 46 (20.5\%) & $16(7.2 \%)$ & $6(2.6 \%)$ & $(2.03-2.29)$ & 2 & $224(100 \%)$ \\
\hline $\begin{array}{l}\text { Al is useful in automated } \\
\text { imaging protocol selection } \\
\text { according to clinical question } \\
\text { and patient condition }\end{array}$ & $\begin{array}{c}46 \\
(20.5 \%)\end{array}$ & $\begin{array}{c}102 \\
(45.6 \%)\end{array}$ & 48 (2।.4\%) & 22 (9.9\%) & $6(2.6 \%)$ & $(2.16-2.42)$ & 2 & $224(100 \%)$ \\
\hline $\begin{array}{l}\text { Al will be useful in improving } \\
\text { diagnosis and saving time }\end{array}$ & 74 (33\%) & $\begin{array}{c}94 \\
(42.1 \%)\end{array}$ & 38 (I6.8\%) & 16 (7.2\%) & 2 (0.9\%) & $(1.89-2.13)$ & 2 & $224(100 \%)$ \\
\hline $\begin{array}{l}\text { Al assists in personalizing } \\
\text { imaging for patients such as } \\
\text { tracking radiation and follow up } \\
\text { examinations }\end{array}$ & $\begin{array}{c}40 \\
(17.9 \%)\end{array}$ & $\begin{array}{c}116 \\
(51.7 \%)\end{array}$ & 40 (I7.9\%) & $24(10.7 \%)$ & 4 (1.8\%) & $(2.14-2.39)$ & 2 & $224(100 \%)$ \\
\hline
\end{tabular}

such as tracking radiation dose and follow-up examinations, respectively.

No significant difference was found in level of agreement among study participants based on profession, academic qualification, years of experience and hospital types $(p>0.05)$.

\section{Discussion}

Diagnostic imaging is clearly undergoing a transformative change with artificial intelligence; therefore, this study was conducted to determine the knowledge and perception of Saudi radiology personnel about AI in the radiological field. On average, $70 \%$ of study participants either agree or strongly agree about the usefulness of the AI-based application in clinical decision making, setting scanning protocols, improving patient care and saving time.

Although AI is being recently introduced in the field of radiology ${ }^{20}$ this study found that most of the participants had a good basic understanding of this technology. It should be noted, however, that there is no objective criterion to determine participants' understanding. Similar findings were found in different studies, ${ }^{18,21}$ which may be due to the fact that AI has become a contentious topic of discussion in radiology. ${ }^{22,23}$ While all the four groups were familiar with the concept of AI in simulating the analytic functions of humans, radiologists tend to be more familiar with this concept than others. Indeed, AI development recently has specifically focused on image interpretation. ${ }^{12}$

However, in this study, radiologists' trust level towards the concept of relying on machine learning ability in analysing data for decision making purposes is relatively limited. A potential reason to this finding might have a relationship to concerns regarding patients' own risk when it comes to AIbased interpretation. ${ }^{4}$ The literature suggests that $\mathrm{AI}$ is not appropriate for the final image interpretation and should remain in the hands of humans, but it can facilitate realtime workflow management as well as urgent exam prioritization, ${ }^{23}$ which both medical students and radiologists endorse. ${ }^{24,25}$ A potential occurrence of $\mathrm{AI}$ errors might raise concerns regarding legal issues, since it is the radiologists' responsibility to notice possible mistakes in AI-based interpretations. ${ }^{26}$ The benefits of AI-based interpretation might greatly diminish if radiologists need to check each interpretation, especially with the surge in images that they have to read. This situation might worsen even with AI support, as the volume and complexity of reports might increase with more data generated in the future.

An understanding of the implications associated with AI is crucial for medical practitioners, especially the meaning of the technology and its contribution to the radiology profession. Experts argue that AI-based applications will change the economic, scientific, clinical and 
ethical future of radiology. ${ }^{22}$ However, there is a counter argument concerning the use of AI-based applications and their positive and/or negative impact on the radiology profession. In a study by Ryan et al, participants reported that AI implementation would lead to a decrease in staffing levels and the creation or integration of new roles. ${ }^{21}$ However, no changes in radiologist staffing level were anticipated by different studies. Not only this, but they also expected an expansion in their roles. ${ }^{1,27}$ In the current study, only one-third of participants think that AI will replace their jobs in the future.

However, almost half of the participated students in this study felt that they are threatened by AI applications. An explanation to this belief could be the absence of subject related to $\mathrm{AI}$ in radiography undergraduate programmes. Therefore, the basic of AI application functions could be misunderstood among students, especially due to the fact that they have never used this application in clinical practice. A similar finding was found in a study that was carried out in the UK, where students reported that they were less likely to consider a career in radiology due to $\mathrm{AI} .^{28}$ It was evident in another study that there is a concern that increasing use of AI technology could result in negative perceptions being drawn towards the professions. ${ }^{21}$ The availability of AI-based applications is limited worldwide, particularly in Saudi Arabia. The use of AI in radiology is unlikely to become widely adopted anytime soon. This is largely due to the high costs associated with training some of these tools independently for each disease and each condition, and also how AI is actually integrated into clinical practice, which is an extremely labour-intensive process. ${ }^{29}$

Almost $95 \%$ of all participants showed interest in AI education. This is mirrored by most participants in previous studies. ${ }^{30,31}$ Therefore, introducing AI education into future curricula has become a must as per most studies' recommendations. ${ }^{20}$ Only $26.8 \%$ of all participants had received education with regard to AI technology, suggesting that AI education is still at early stages, especially when knowing that this education is a self-effort as to the authors' knowledge, no formal education is being provided to radiology personnel at any level of their programmes. ${ }^{20}$ Overall willingness in implementing AI technology into clinical practice was shown by all participants, which was consistent with the literature where positive attitudes towards AI implementations in radiological field were shown. ${ }^{20,32}$ The radiology community must, in fact, extend great effort to ensure AI's future role in this field is well supported. Training and education programmes should be established to teach radiology personnel how to use AI-based applications in their clinical practice. Not only this, but they also should be involved in $\mathrm{AI}$ development process.

\section{Limitations}

This study was designed to investigate Saudi radiology personnel's perceptions of Artificial Intelligence implementation in radiology department, and the findings may need to be interpreted with caution due to the small size of the sample, and consequently may not be generalised to the whole radiology population in Saudi Arabia. Another limitation of this study was sampling bias, online survey might be restricted to those who are more active online, especially those who have social media accounts (WhatsApp, Twitter); however, survey was distributed via various online channels to improve its visibility among participants such as emails and messages.

\section{Conclusion}

Evaluating the knowledge of radiology personnel about AI application is of great importance as this technology has started to filter into clinical departments. The findings of this study showed overall positive attitude to AI and good knowledge base was shown by them. However, some participants felt that their jobs are threatened by this technology and this belief could change if good training and education programmes were designed to improve their understanding about AI application. A conclusion to the debate on whether AI will take over radiology personnel has not been drawn yet until this technology is fully implemented into clinical departments.

\section{Disclosure}

The authors report no conflicts of interest in this work.

\section{References}

1. Lee LI, Kanthasamy S, Ayyalaraju RS, Ganatra R. The current state of artificial intelligence in medical imaging and nuclear medicine. BJR Open. 2019;1:20190037.

2. Royal College of Radiologists. Clinical Radiology UK Workforce Census 2015 Report; 2016.

3. Bin Dahmash A, Alabdulkareem M, Alfutais A, et al. Artificial intelligence in radiology: does it impact medical students preference for radiology as their future career? BJR Open. 2020;2:20200037.

4. European Society of Radiology (ESR) communications@ myesr. org Emanuele Neri Nandita de Souza Adrian Brady Angel Alberich Bayarri Christoph D. Becker Francesca Coppola Jacob Visser. What the radiologist should know about artificial intelligence - an ESR white paper. Insights Imaging. 2019;10:1-8. 
5. Kohli M, Geis R. Ethics, artificial intelligence, and radiology. $J$ Am Coll Radiol. 2018;15(9):1317-1319. doi:10.1016/j.jacr.2018.05.020

6. Martini K, Blüthgen C, Eberhard M, et al. Impact of vessel suppressed-CT on diagnostic accuracy in detection of pulmonary metastasis and reading time. Acad Radiol. 2021;28(7):988-994. doi:10.1016/j.acra.2020.01.014

7. Kim H, Park CM, Hwang EJ, Ahn SY, Goo JM. Pulmonary subsolid nodules: value of semi-automatic measurement in diagnostic accuracy, diagnostic reproducibility and nodule classification agreement Eur Radiol. 2018;28(5):2124-2133. doi:10.1007/s00330-017-5171-7

8. Martin DD, Deusch D, Schweizer R, Binder G, Thodberg HH, Ranke MB. Clinical application of automated greulich-pyle bone age determination in children with short stature. Pediatr Radiol. 2009;39(6):598-607. doi:10.1007/s00247-008-1114-4

9. Nehrer S, Ljuhar R, Steindl P, et al. Automated knee osteoarthritis assessment increases physicians' agreement rate and accuracy: data from the osteoarthritis initiative. Cartilage. 2019;1947603519888793. doi:10.1177/1947603519888793

10. Hosny A, Parmar C, Quackenbush J, Schwartz LH, Aerts HJ. Artificial intelligence in radiology. Nat Rev Cancer. 2018;18 (8):500-510.

11. Alsharif W, Qurashi A. Effectiveness of COVID-19 diagnosis and management tools: a review. Radiography. 2021;27(2):682-687. doi:10.1016/j.radi.2020.09.010

12. Hardy M, Harvey H. Artificial intelligence in diagnostic imaging: impact on the radiography profession. $B r \quad J$ Radiol. 2020;93 (1108):20190840. doi:10.1259/bjr.20190840

13. Pongnapang N. Practical guidelines for radiographers to improve computed radiography image quality. Biomed Imaging Interv $J$. 2005;1(2). doi:10.2349/biij.1.2.e12

14. Beetz M, Buss M, Wollherr D. Cognitive Technical Systems-What is the Role of Artificial Intelligence? Springer; 2007:19-42.

15. Mehrizi MHR, van Ooijen P, Homan M. Applications of artificial intelligence (AI) in diagnostic radiology: a technography study. Eur Radiol. 2021;31(4):1805-1811. doi:10.1007/s00330-020-07230-9

16. Li J, Zhou Z, Dong J, et al. Predicting breast cancer 5-year survival using machine learning: a systematic review. PLoS One. 2021;16(4): e0250370. doi:10.1371/journal.pone.0250370

17. Noguerol TM, Paulano-Godino F, Martín-Valdivia MT, Menias CO, Luna A. Strengths, weaknesses, opportunities, and threats analysis of artificial intelligence and machine learning applications in radiology. J Am Coll Radiol. 2019;16(9):1239-1247. doi:10.1016/j.jacr.2019.05.047

18. Gong B, Nugent JP, Guest W, et al. Influence of artificial intelligence on Canadian medical students' preference for radiology specialty: a national survey study. Acad Radiol. 2019;26(4):566-577. doi:10.1016/j.acra.2018.10.007

19. van Hoek J, Huber A, Leichtle A, et al. A survey on the future of radiology among radiologists, medical students and surgeons: students and surgeons tend to be more skeptical about artificial intelligence and radiologists may fear that other disciplines take over. Eur J Radiol. 2019;121:108742. doi:10.1016/j.ejrad.2019.108742
20. Tajaldeen A, Alghamdi S. Evaluation of radiologist's knowledge about the artificial intelligence in diagnostic radiology: a surveybased study. Acta Radiol Open. 2020;9(7):2058460120945320. doi:10.1177/2058460120945320

21. Ryan M, O’Donovan T, McNulty JP. Artificial intelligence: the opinions of radiographers and radiation therapists in Ireland. Radiography. 2021;27:S74-S82. doi:10.1016/j.radi.2021.07.022

22. Jungmann F, Jorg T, Hahn F, et al. Attitudes toward artificial intelligence among radiologists, IT specialists, and industry. Acad Radiol. 2021;28(6):834-840. doi:10.1016/j.acra.2020.04.011

23. Holdsworth C, Kukluk J, Molodowitch C, et al. Computerized system for safety verification of external beam radiation therapy planning. Int J Radiat Oncol Biol Phys. 2017;98(3):691-698. doi:10.1016/j. ijrobp.2017.03.001

24. Coppola F, Faggioni L, Regge D, et al. Artificial intelligence: radiologists' expectations and opinions gleaned from a nationwide online survey. Radiol Med. 2021;126(1):63-71. doi:10.1007/s11547-02001205-y

25. Dos Santos DP, Giese D, Brodehl S, et al. Medical students' attitude towards artificial intelligence: a multicentre survey. Eur Radiol. 2019;29(4):1640-1646. doi:10.1007/s00330-018-5601-1

26. Smith MJ, Bean S. AI and ethics in medical radiation sciences. $J$ Med Imaging Radiat Sci. 2019;50(4):S24-S26. doi:10.1016/j. jmir.2019.08.005

27. Waymel Q, Badr S, Demondion X, Cotten A, Jacques T. Impact of the rise of artificial intelligence in radiology: what do radiologists think? Diagn Interv Imaging. 2019;100(6):327-336. doi:10.1016/j. diii.2019.03.015

28. Sit C, Srinivasan R, Amlani A, et al. Attitudes and perceptions of UK medical students towards artificial intelligence and radiology: a multicentre survey. Insights Imaging. 2020;11(1):1-6. doi:10.1186/s13244-019-0830-7

29. Summers RM. Progress in fully automated abdominal CT interpretation. Am J Roentgenol. 2016;207(1):67-79. doi:10.2214/ AJR.15.15996

30. Liang S, Tang F, Huang X, et al. Deep-learning-based detection and segmentation of organs at risk in nasopharyngeal carcinoma computed tomographic images for radiotherapy planning. Eur Radiol. 2019;29(4):1961-1967. doi:10.1007/s00330-018-5748-9

31. Wong K, Gallant F, Szumacher E. Perceptions of Canadian radiation oncologists, radiation physicists, radiation therapists and radiation trainees about the impact of artificial intelligence in radiation oncology-national survey. J Med Imaging Radiat Sci. 2021;52(1):44-48. doi:10.1016/j.jmir.2020.11.013

32. El Naqa I, Haider MA, Giger ML, Ten Haken RK. Artificial intelligence: reshaping the practice of radiological sciences in the 21 st century. $B r \quad J$ Radiol. 2020;93(1106):20190855. doi:10.1259/ bjr.20190855
Journal of Multidisciplinary Healthcare

\section{Publish your work in this journal}

The Journal of Multidisciplinary Healthcare is an international, peerreviewed open-access journal that aims to represent and publish research in healthcare areas delivered by practitioners of different disciplines. This includes studies and reviews conducted by multidisciplinary teams as well as research which evaluates the results or conduct of such teams or healthcare processes in general. The journal covers a very wide range of areas and welcomes submissions from practitioners at all levels, from all over the world. The manuscript management system is completely online and includes a very quick and fair peer-review system. Visit http://www.dovepress.com/testimonials. php to read real quotes from published authors. 